\title{
Prevención de recaídas en la esquizofrenia: Propuesta de un programa de intervención durante la fase prodrómica
}

\author{
Juan Francisco Godoy García ${ }^{1,2}$, María Caballero Martínez ${ }^{1}$, Débora Godoy-Izquierdo ${ }^{1,2}, M^{a}$ \\ Luisa Vázquez Pérez ${ }^{1,3}$ y José A. Muela Martínez ${ }^{4}$ \\ ${ }^{1}$ Grupo de Investigación Psicología de la Salud y Medicina Conductual (CTS-267). Universidad de Granada \\ ${ }^{2}$ Departamento de Personalidad, Evaluación y Tratamiento Psicológico. Universidad de Granada \\ ${ }^{3}$ Departamento de Pedagogía y Psicología. Centro Universitario SAFA. Universidad de Jaén \\ ${ }^{4}$ Departamento de Psicología. Universidad de Jaén \\ Recepción: 4 de abril de 2016 | Revisión: 5 de abril de 2016 | Aceptado: 11 abril de 2016 \\ Correspondencia: jgodoy@ugr.es \\ Citar: Godoy García, JF., Caballero Martínez, M., Godoy-Izquierdo, D., Vázquez Pérez, ML. y Muela Martínez, JA. (2016). Prevención \\ de recaídas en la esquizofrenia: Propuesta de un programa de intervención durante la fase prodrómica. ReiDoCrea, 5, 56-68.
}

Agradecimientos: Esta investigación fue realizada con la ayuda financiera suministrada al grupo de investigación "Psicología de la Salud \& Medicina Conductual" (CTS-0267) por la Consejería de Innovación, Ciencia y Empresa, Junta de Andalucía (España)

Resumen: La esquizofrenia es un trastorno psicológico crónico y grave que afecta al 1\% de la población mundial adulta. El curso del pródromo comienza en la mayoría de los casos con la experimentación de síntomas clínicos negativos, seguidos de síntomas positivos, que se van agravando en frecuencia, duración e intensidad conforme se aproxima el episodio de psicosis. Se propone una intervención multicomponente destinada a pacientes con esquizofrenia y a sus familiares, con 24 sesiones dirigidas a la psicoeducación y adherencia al tratamiento, detección e intervención sobre signos tempranos y fortalecimiento de la autoeficacia. Dicha intervención pretende ayudarles a detectar y modificar los principales marcadores prodrómicos, entrenando a los pacientes y a sus familiares en habilidades útiles para prevenir o afrontar la recaída, con el fin de evitar o retrasar la nueva crisis psicótica. Consecuentemente, cabe esperar que los pacientes mejoren la comprensión de su enfermedad y su adherencia a la medicación, reconozcan y actúen sobre los primeros signos de la posible recaída, incrementen su autoeficacia, se reduzcan las tasas de recaída y, en definitiva, mejore tanto su estado clínico como su calidad de vida. En cuanto a los familiares, se persigue, por un lado, ayudar al paciente en todo lo anterior y, por otro, aliviar su carga como cuidadores, mejorando su calidad de vida.

Palabras clave: Psicología clínica | Esquizofrenia

Relapse Prevention in Schizophrenia: A Proposal of Intervention during the Prodromal Phase

Abstract: Schizophrenia is a chronic, severe mental disorder affecting $1 \%$ of the adult population worldwide (WHO, 2014). The course of the prodromal stage generally begins with experiencing negative clinical symptoms, followed by positive symptoms, which usually increase in frequency, duration and intensity as the episode of psychosis approaches. A 24-session multi-component intervention -psychoeducation and adherence to treatment, early detection and intervention on initial signs and self-efficacy strengthening- is proposed herein, which is aimed at patients with schizophrenia and their relatives. The intervention aims to help them in detecting and modifying the main prodromal markers by enhancing patients' and relatives' skills for preventing or managing a relapse, in order to avoid or delay the new psychotic crisis. Consequently, it can be expected that patients' understanding of their disease and their medication adherence will improve, as well as their capability for recognizing and acting on the first signs of a possible relapse and their self-efficacy expectations. It can be also expected that relapse rates will be reduced and, ultimately, that patients' clinical status and quality of life will be enhanced. Relatives are expected to show enhanced capability for helping the patient in all the above and, as a consequence, their disease-related burden as caregivers will be reduced and their quality of life improved.

Keywords: Clinical psychology | Schizophrenia

\section{Introducción}

La esquizofrenia es un trastorno mental crónico grave caracterizado por una amplia gama de comportamientos inusuales que incluyen la distorsión del pensamiento, las percepciones, las emociones, el lenguaje, la conciencia de sí mismo y la conducta (OMS, 2014). La principal consecuencia es una profunda alteración tanto en la vida de las personas que sufren la enfermedad como de quienes les rodean (Britto, 2014). Los criterios diagnóstico para la esquizofrenia, según el DSM-V (APA, 2014), se presentan en la Tabla 1. 


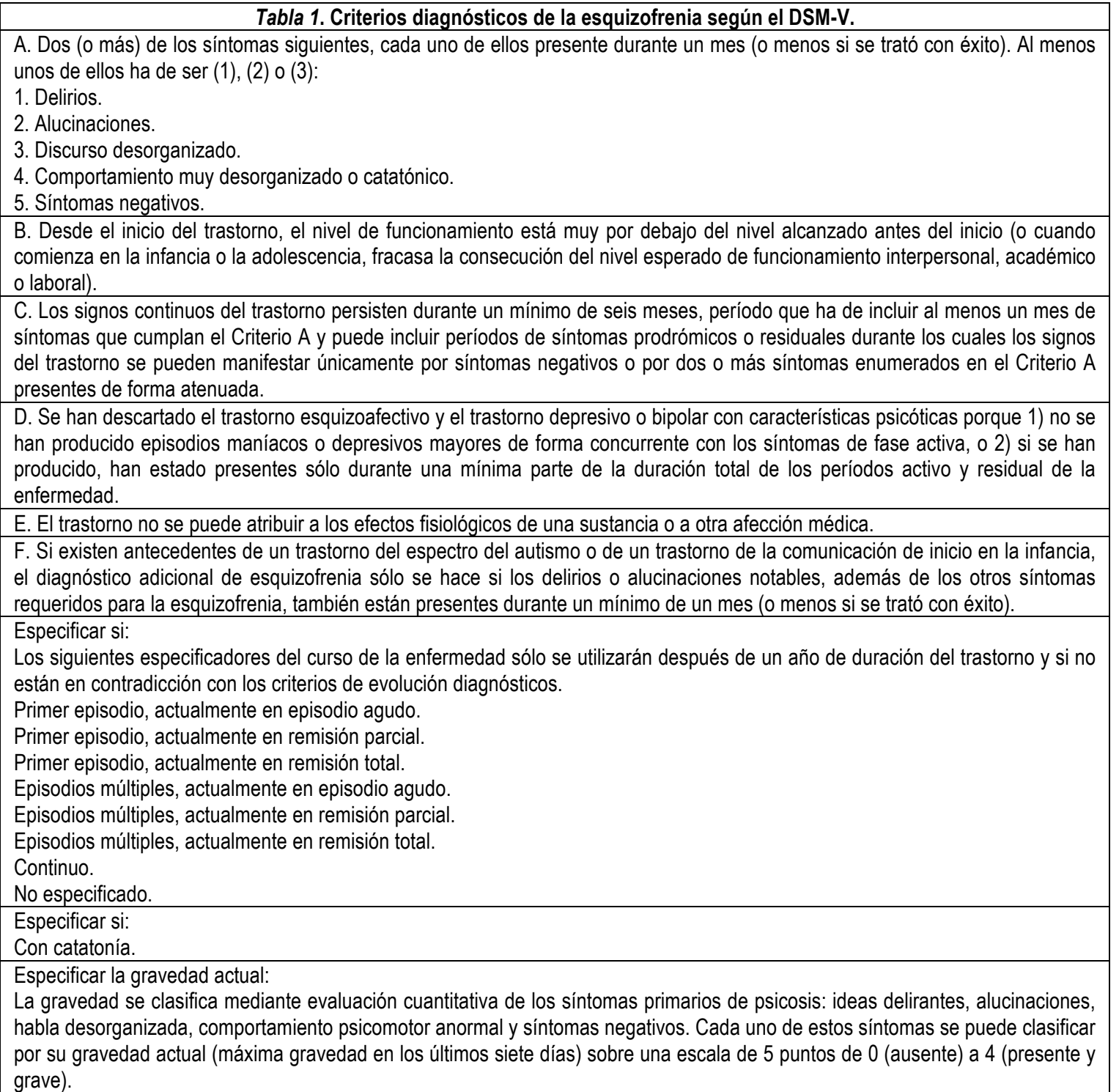

Aunque existe una amplia investigación sobre la esquizofrenia, aún se desconoce su causa. Actualmente, afecta aproximadamente a 21 millones de personas, esto es, al $1 \%$ de la población mundial adulta, siendo más frecuente y precoz en hombres que en mujeres (OMS, 2014). Estas cifras reflejan el alto impacto personal, familiar y económico de la esquizofrenia. Godoy, Muela y Godoy-Izquierdo (2012) estiman que

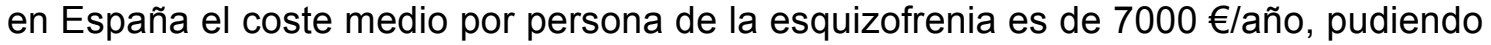
triplicarse si los pacientes recaen (Ascher-Svanum et al., 2010).

En referencia a las intervenciones terapéuticas farmacológicas, las formulaciones de acción prolongada y los antipsicóticos atípicos parecen ser preferibles a los convencionales (Larson, Walker y Compton, 2010; Samalin, Blanc y Llorca, 2010). También se ha encontrado que la medicación antidepresiva mejora la sintomatología en fase prodrómica (Larson et al., 2010). Sin embargo, la medicación antipsicótica plantea tres importantes problemas: primero, se asocia con efectos secundarios significativos, que en algunos casos pueden ser muy graves, como el síndrome metabólico (Álvarez-Jiménez et al., 2012; Patel et al., 2009); segundo, no parece ser eficaz para los síntomas negativos, los déficit cognitivos y el deterioro del funcionamiento cotidiano (APA-SCP, 2013); y tercero, frecuentemente estos pacientes no se adhieren al tratamiento, lo que cuadruplica el riesgo de recaída (ÁlvarezJiménez et al., 2012). 
Consecuentemente, es necesario llevar a cabo intervenciones psicológicas y psicosociales (APA-SCP, 2013). Las que cuentan con un apoyo empírico robusto son la terapia cognitivo-conductual (TCC), el entrenamiento en habilidades sociales, el tratamiento asertivo comunitario, la remediación cognitiva, la psicoeducación familiar, el apoyo laboral y vital, los programas de manejo y recuperación de la enfermedad, las intervenciones en el primer episodio de psicosis y durante la fase prodrómica, las intervenciones sobre estilos de vida saludables y los grupos de ayuda mutua (APASCP, 2013; Mueser, Deavers, Penn y Cassisi, 2013). Éstas mejoran las estrategias de afrontamiento, conductas, emociones y pensamientos, reducen los síntomas positivos y negativos, aumentan el control sobre los síntomas y disminuyen su gravedad, mejorando el ajuste y funcionamiento psicosocial del paciente, su calidad de vida, la carga familiar y la adherencia al tratamiento (Larson et al., 2010; Olivares, Sermon, Hemels y Schreiner, 2013). No obstante, ningún tratamiento es eficaz para la curación de la esquizofrenia, por lo que su curso normal se define por la cronicidad y la alternancia de períodos de remisión y recaídas (Godoy, Muela et al., 2012).

El inicio de la primera crisis y de la recaída puede estar precedido por alteraciones en la cognición, la emoción, la percepción, la conducta, la comunicación, la motivación y el sueño, de menor a mayor gravedad hasta que se transforman en la crisis propiamente dicha (Larson et al., 2010). Godoy (2001, citado en Godoy, Muela et al., 2012) define la recaída psicótica como "un deterioro en el estado clínico caracterizado por la exacerbación de síntomas psicóticos que conlleva la rehospitalización del paciente o un importante cambio en el tratamiento antipsicótico", algo que ocurre aproximadamente en el $50 \%$ de los pacientes en remisión.

El curso del pródromo, período variable en duración previo a la primera crisis o la recaída, comienza con la experimentación de síntomas clínicos negativos -depresión, ansiedad, anhedonia, déficit cognitivos, aislamiento, problemas en el funcionamiento cotidiano, etc.-, seguido de síntomas positivos -delirios, alucinaciones, trastornos del habla, pensamiento desordenado, etc.-, que se van agravando en términos de frecuencia, duración, intensidad y grado de interferencia con el funcionamiento cotidiano del paciente conforme se aproxima el episodio de psicosis (Larson et al., 2010). En su estudio pionero al respecto, Godoy, Muela, Sánchez-Barrera, SánchezHuete, Pérez y Lorite (1995) identificaron como pródromos o marcadores de la recaída los recogidos en las Tablas 2 y 3 .

Tabla 2. Síntomas prodrómicos informados por los propios pacientes. Extraído de Godoy et al. (1995).

\begin{tabular}{|cl|}
\hline $\begin{array}{c}\text { Días previos } \\
\text { a la recaída }\end{array}$ & Síntomas \\
\hline 60 & Conductas raras o extrañas. \\
\hline 45 & $\begin{array}{l}\text { Soledad, distanciamiento de familiares y amigos, conducta desinhibida, agresiva, colérica o } \\
\text { violenta y excesiva preocupación por cosas o personas. }\end{array}$ \\
\hline 38 & Problemas de atención o concentración. \\
\hline 30 & Ensimismamiento, retraimiento, aislamiento, irritabilidad, nerviosismo y agitación. \\
\hline 22 & Tensión, miedo, ansiedad. \\
\hline 20 & Sensaciones corporales inusuales o extrañas. \\
\hline 18 & Sentimientos inapropiados. \\
\hline 17 & Pesimismo y desesperación. \\
\hline 14 & Sensaciones visuales o auditivas inusuales o extrañas. \\
\hline 7 & Desinterés, apatía y aburrimiento \\
\hline 6 & Problemas de sueño. \\
\hline 5 & Confusión, desorientación, depresión y tristeza. \\
\hline
\end{tabular}




\begin{tabular}{|cl|}
\hline \multicolumn{2}{|c|}{ Tabla 3. Síntomas prodrómicos informados por los familiares. Extraído de Godoy et al. (1995). } \\
\hline $\begin{array}{c}\text { Días previos } \\
\text { a la recaída }\end{array}$ & Síntomas \\
\hline 14 & Conductas raras o extrañas. \\
\hline 10 & Desinterés, apatía y aburrimiento. \\
\hline 7 & Beber o fumar excesivamente e irritabilidad. \\
\hline 4 & Fatiga, cansancio y conducta tímida e inhibida. \\
\hline 3 & Ensimismamiento, retraimiento, aislamiento, depresión y tristeza. \\
\hline
\end{tabular}

Consecuentemente, la identificación precoz de las personas con alto riesgo de desarrollar un trastorno psicótico o una recaída supone una gran oportunidad de intervención antes del desarrollo de la enfermedad o la nueva crisis psicótica, lo que repercute positivamente en el pronóstico de estos pacientes (Larson et al., 2010). Existe una creciente evidencia de que los programas centrados en el primer episodio de psicosis ofrecen los mejores resultados (Mueser y Cook, 2014). La prevención de recaídas es una novedosa e importante línea de actuación destinada a mejorar la evolución del trastorno (Godoy, Muela et al., 2012), siendo las intervenciones durante la fase prodrómica señaladas de gran utilidad clínica (Mueser et al., 2013).

Por ello, en este trabajo se propone una intervención destinada a la prevención de recaídas en la esquizofrenia, centrada en la detección y modificación de los principales marcadores prodrómicos con el fin de evitar, o retrasar al máximo, la nueva crisis psicótica en pacientes diagnosticados previamente de esquizofrenia.

\section{"Desafiando a la irrealidad": Programa de intervención durante la fase prodrómica para la prevención de recaídas en la esquizofrenia}

El programa "Desafiando a la irrealidad" consta de 24 sesiones, distribuidas durante 6 meses, a razón de una sesión semanal de dos horas de duración. La intervención está destinada tanto a pacientes diagnosticados de esquizofrenia como a sus familiares, y consta de tres componentes: psicoeducación y adherencia al tratamiento (Godoy, Godoy-Izquierdo y Vázquez, 2012; Prasko, Vrbova, Latalova y Mainerova, 2011; Samalin et al., 2010), detección e intervención sobre signos tempranos, básicamente mediante TCC (APA-SCP, 2013; Robles, Páez y González, 2012; Shean, 2007; Valencia, Liberman, Rascón y Juárez, 2012) y fortalecimiento de la autoeficacia (Godoy, Godoy-Izquierdo et al., 2012). A continuación se detallan las sesiones (numeradas según su orden de aplicación) y contenidos específicos del programa y de cada sesión.

\section{Presentación del programa:}

- Introducción de pacientes y familiares en el contexto de la intervención, presentándose terapeutas, pacientes, el programa en sí y los logros que se pretenden alcanzar, esto es, dotar al paciente y sus cuidadores de recursos eficaces para mejorar su desarrollo personal, funcionamiento psicosocial y calidad de vida y reducir el número de recaídas y la gravedad de las mismas en caso de que se produzcan.

- Componente psicoeducativo y de adherencia al tratamiento: Información sobre qué es la esquizofrenia, su prevalencia en nuestra comunidad, cuáles son sus causas, sus síntomas típicos, el impacto de dicha sintomatología, cómo puede ayudar la familia, pros y contras de la medicación, repercusiones de una adherencia inadecuada a la medicación y su relación con las recaídas. Se 
enfatiza la necesidad de implicación en el programa y la posibilidad de preguntar dudas o inquietudes en cualquier momento.

- Inventario de Actitudes al Medicamento (DAI) de Hogan, Awad y Eastwood (1983) y explicación de cómo rellenar la hoja de registro de medicación para su registro diario en casa, controlándose de forma continuada la toma de medicación.

\section{Reconocimiento de las señales de alerta de una recaída:}

- Revisión de tareas para casa, resolviendo los problemas surgidos y utilizando el refuerzo positivo y las instrucciones correctivas de mejora.

- Componente psicoeducativo y de adherencia al tratamiento: Descripción del modelo de vulnerabilidad-estrés (Zubin y Spring, 1977), reconocimiento de los factores desencadenantes y de mantenimiento de la recaída y establecimiento del orden cronológico de aparición de los síntomas prodrómicos. Se entrega una tabla resumen con los principales síntomas del pródromo y el tiempo de aparición medio previo a la recaída.

- Componente de autoeficacia: Explicación de qué es la autoeficacia, su importancia conductual, las fuentes de autoeficacia y el papel que juega en la recaída.

- Explicación de cómo rellenar el autorregistro de pensamientos positivos y negativos, para que los pacientes lo rellenen a diario en casa -junto con el registro diario de medicación- y aprendan a identificar y evaluar las consecuencias de sus pensamientos.

\section{Acercamiento a la prevención de recaídas:}

- Corrección de tareas para casa.

- Componente psicoeducativo y de adherencia al tratamiento: Información sobre los estilos de vida, el manejo del estrés, el control de la respiración y relajación, la gestión del tiempo y la realización de actividades placenteras.

- Componente de intervención sobre signos tempranos: Se enseña a eliminar las contingencias positivas tras las conductas inadecuadas de los pacientes y a reforzar las conductas funcionales.

- Componente de autoeficacia: Se enfatiza la necesidad de actuar ante las dificultades, incrementando la motivación y la capacidad personal para resolver problemas, identificando y anticipando los efectos negativos de no actuar.

- Explicación de cómo rellenar el autorregistro de conductas, para que los pacientes las registren semanalmente en casa -junto con el registro diario de medicación y el autorregistro de pensamientos, en adelante, tareas para casa-.

\section{Control de conductas raras o extrañas:}

- Corrección de tareas para casa.

- Componente de intervención sobre signos tempranos: Se enseña a reconocer e intervenir sobre las conductas raras o extrañas. Se expone el modelo ABC para que los pacientes rellenen un autorregistro que les permita detectar los antecedentes y consecuentes de sus pensamientos, emociones y conductas disfuncionales. Se enseñan habilidades sociales básicas que faciliten la adaptación al medio.

- Componente de autoeficacia: Se realiza un entrenamiento en autocontrol, enseñando el control estimular, las autoinstrucciones y el autorrefuerzo.

- Tareas para casa. 
5. Control de síntomas de soledad y distanciamiento de familiares y amigos:

- Corrección de tareas para casa.

- Componente de intervención sobre signos tempranos: Se enseñan habilidades de comunicación y a establecer/mantener relaciones sociales positivas, mediante modelado y moldeado, role-playing y reforzamiento social positivo.

- Componente de autoeficacia: Se realiza un entrenamiento en afrontamiento de problemas de comunicación y habilidades sociales, presentando vídeos de personas interaccionando para que identifiquen qué conductas son adecuadas, cuáles no y cómo pueden solucionarse. Se practica lo aprendido mediante ensayos conductuales.

- Tareas para casa.

6. Control de síntomas violentos, agresivos y de desinhibición:

- Corrección de tareas para casa.

- Componente de intervención sobre signos tempranos: Se realiza un árbol colaborativo que incite a los pacientes a reflexionar sobre una situación, sus pensamientos, sentimientos y necesidades, las estrategias que ponen en marcha y cómo podrían mejorar. El grupo ofrece retroalimentación sobre el modo de actuar de sus compañeros. Se enseñan técnicas de relajación guiada en imaginación, respiración profunda y relajación muscular progresiva de Jacobson (1929).

- Componente de autoeficacia: Se continúa el entrenamiento en autocontrol, iniciado en la Sesión 4.

- Tareas para casa.

7. Control de síntomas de preocupación excesiva y problemas de atención o concentración:

- Corrección de tareas para casa.

- Componente de intervención sobre signos tempranos: Se realiza un entrenamiento en gestión del tiempo, incluyendo la participación diaria en actividades placenteras. Se enseña a detener el pensamiento cuando éste comienza a ser redundante y disfuncional y a modificar las creencias negativas asociadas, realizando reestructuración cognitiva mediante una demostración práctica.

- Componente de autoeficacia: Se realiza un entrenamiento cognitivo, minimizando los pensamientos negativos y potenciando los positivos. Se enseñan estrategias de resolución de problemas.

- Tareas para casa.

8. Control de síntomas de irritabilidad, nerviosismo y agitación:

- Corrección de tareas para casa.

- Componente de intervención sobre signos tempranos: Se repasan las técnicas de relajación trabajadas en la Sesión 6 y se enseñan otras más adaptadas a las necesidades y experiencias de cada participante, si fuera necesario.

- Componente de autoeficacia: Se trabaja el afrontamiento al estrés, anotando cada paciente una lista de estresores cotidianos, los pensamientos y sentimientos que éstos les evocan y las respuestas que suelen ofrecer. Se les enseñan diferentes estrategias de afrontamiento al estrés.

- Tareas para casa. 
9. Control de síntomas de tensión, miedo y ansiedad:

- Corrección de tareas para casa.

- Componente de intervención sobre signos tempranos: Se realiza un roleplaying que potencia las relaciones personales, pues pacientes y familiares deben interactuar positivamente, compartiendo sus emociones y experiencias y mejorando el apoyo social en condiciones de malestar emocional. Se repasan las competencias entrenadas en sesiones anteriores para aplicarlas a la autorregulación emocional.

- Componente de autoeficacia: Se continúa con el manejo de síntomas y se retoma el entrenamiento en autocontrol.

- Tareas para casa.

10. Control de sensaciones corporales inusuales o extrañas:

- Corrección de tareas para casa.

- Componente de intervención sobre signos tempranos: Se reanuda el trabajo con el modelo $A B C$ (Sesión 4), para que los pacientes rellenen el autorregistro cada vez que experimenten sensaciones extrañas, anotando lo que sienten, piensan y hacen antes y después de que la sensación aparezca, determinando qué conductas deben desaparecer o cuáles otras potenciar en sus rutinas. Se realiza reestructuración cognitiva mediante el esquema ABCDE, para cuestionar la veracidad y efectos de sus experiencias tras una prueba de realidad. Se retoman los contenidos de habilidades de comunicación para que expresen adecuadamente sus sensaciones y experiencias.

- Componente de autoeficacia: Se enseña a afrontar los síntomas operativizándolos e implantando respuestas deseables mediante la autoevaluación, el autorrefuerzo y la autocorrección.

- Tareas para casa y autorregistro ABCDE.

11. Control de sentimientos inapropiados:

- Corrección de tareas para casa.

- Componente de intervención de signos tempranos: Se desarrolla de forma similar a la sesión anterior, pero centrándose en los sentimientos experimentados. Se entrega un listado de sentimientos y emociones para facilitar su expresión.

- Componente de autoeficacia: Se aborda de manera similar a la sesión anterior.

- Tareas para casa.

12. Adherencia a la medicación 1:

- Corrección de tareas para casa.

- Componente psicoeducativo y de adherencia al tratamiento: Se repasan los contenidos y técnicas abordadas hasta el momento, resolviendo las dudas y dificultades encontradas. Se consulta con los familiares los cambios percibidos en los pacientes y en sí mismos respecto al modo de manejar la enfermedad. Aunque los pacientes rellenan diariamente un autorregistro de medicación, se vuelve a pasar el DAl para verificar si ha mejorado la adherencia a la medicación o si, por el contrario, se debe incidir en ello.

- Componente de autoeficacia: Se enfatiza la percepción de controlabilidad sobre la enfermedad a través del uso adecuado de la medicación y el resto de recursos psicosociales aprendidos. 
- Tareas para casa.

13. Adherencia a la medicación 2:

- Componente psicoeducativo y de adherencia al tratamiento: Basada en la propuesta de Safren (2000, citado en Robles et al., 2012), la sesión pretende aumentar la credibilidad, confianza y expectativas del tratamiento, debatiendo sobre las ventajas de tomar ininterrumpidamente la medicación frente a los efectos secundarios de la misma y los efectos del abandono de la misma.

- Componente de intervención sobre signos tempranos: Se pretende conseguir un pensamiento adaptativo y la eliminación de las conductas disruptivas mediante TCC. Se pone en práctica lo aprendido con role-playing en los que intervienen pacientes y familiares.

- Componente de autoeficacia: Se aborda de manera similar a la sesión anterior.

- Tareas para casa.

14. Control de síntomas de pesimismo y desesperación:

- Corrección de tareas para casa.

- Componente de intervención sobre signos tempranos: Se realiza un entrenamiento en autorregulación emocional, para que los pacientes manejen las emociones negativas. Se practican las técnicas de relajación y respiración abordadas en la Sesión 8, la detención del pensamiento y la modificación de las creencias negativas, mediante reestructuración cognitiva, como se hizo en la Sesión 7.

- Componente de autoeficacia: Se trabaja el afrontamiento al estrés, como se abordó en la Sesión 8.

- Tareas para casa.

15. Control de sensaciones visuales o auditivas inusuales:

- Corrección de tareas para casa.

- Componente de intervención sobre signos tempranos: Para trabajar los delirios, se entrega un listado de los más frecuentes y se abordan sus antecedentes con el modelo $A B C$, estableciendo la relación con las emociones disfuncionales. Se realiza modificación cognitiva, debatiendo sobre el grado de convicción y preocupación para plantear después evidencias a favor y en contra del delirio. Se enseña a realizar experimentos conductuales que prueben esas creencias y a extraer conclusiones. Para trabajar las alucinaciones, se sigue el mismo procedimiento, comenzando por las creencias más débiles y continuando con las más rígidas.

- Componente de autoeficacia: Con el registro $A B C$ anterior, se explica que la interpretación que los pacientes hacen de los delirios y las alucinaciones repercute sobre sus conductas y emociones. Se ofrecen como estrategias de afrontamiento las autoinstrucciones, pensamientos positivos, restricción y desviación de la atención, participación en actividades gratificantes y modificación de creencias.

- Tareas para casa.

16. Control de síntomas de desinterés, apatía y aburrimiento:

- Corrección de tareas para casa. 
- Componente de intervención sobre signos tempranos: Los pacientes escriben un listado de actividades de ocio y se les incita a que participen en ellas. Individualmente leen en voz alta sus listados y entre todos aportan ideas. Se abordan las habilidades necesarias para trabajar exitosamente, contribuyendo a la normalización, adaptación y autonomía de los pacientes. En este taller de empleo se informa sobre cómo buscar trabajo, cómo conocer el funcionamiento de cada puesto, cómo resolver los problemas laborales y cómo prevenirlos.

- Componente de autoeficacia: Se realiza un entrenamiento en resolución de problemas similar al de la Sesión 3 y un entrenamiento en conductas de autocuidado.

- Tareas para casa.

17. Control de los problemas en el sueño:

- Corrección de tareas para casa.

- Componente de intervención sobre signos tempranos: Retomando el modelo $A B C$, se establecen las conductas antecesoras a la de irse a la cama que pueden perturbar el sueño. Se explica la importancia de no consumir bebidas estimulantes, hacer ejercicio físico intenso, ingerir comidas copiosas, realizar actividades activadoras, etc. antes de ir a dormir. Se recomienda que antes de dormir se apliquen técnicas de relajación. Se recuerda la detención del pensamiento y la modificación de los pensamientos negativos y su sustitución por otros positivos más funcionales.

- Componente de autoeficacia: Se enseñan nuevas estrategias de afrontamiento al estrés.

- Tareas para casa.

18. Control de síntomas de confusión y desorientación:

- Corrección de tareas para casa.

- Componente de intervención sobre signos tempranos: Se propone escribir un diario, pues mejora la orientación espacial y temporal, permitiendo hacer pruebas de realidad que sometan a juicio las falsas creencias o las experiencias inusuales. Los familiares deben supervisarlo para verificar que refleje los aspectos más importantes de cada día. Se realiza un role-playing en el que se pregunta por la ruta de lugares que los pacientes conocen, obteniendo una medida sobre el grado de confusión y desorientación. Se repasan las habilidades de comunicación, para que expresen adecuadamente lo que sienten y mejoren sus vínculos afectivos así como el apoyo social dado y recibido.

- Componente de autoeficacia: Se continua enseñando a afrontar los síntomas mediante la autoevaluación, las autoinstrucciones y la autocorrección.

- Tareas para casa.

19. Control de síntomas depresivos y tristeza:

- Corrección de tareas para casa.

- Componente de intervención sobre signos tempranos: Utilizando el listado de actividades de ocio de la Sesión 16, se les incita a realizar un autorregistro $\mathrm{ABC}$ tanto si se involucran en ellas como si no, determinando las diferencias emocionales en ambos casos. Se potencian sus relaciones personales, evitando los sentimientos de soledad y las emociones negativas. 
- Componente de autoeficacia: Se trabajan las habilidades de autocontrol, las autoverbalizaciones positivas y el autorrefuerzo. Se debate sobre el autoconcepto, la autoestima y la autoeficacia.

- Tareas para casa.

20. Repaso de los síntomas prodrómicos 1:

- Corrección de tareas para casa.

- Componente psicoeducativo y de adherencia al tratamiento: Se repasan los contenidos y técnicas abordadas durante el programa, utilizando la tabla resumen de la Sesión 2. Se resuelven las dudas y dificultades encontradas por los pacientes y familiares.

21. Repaso de los síntomas prodrómicos 2:

- Componente psicoeducativo y de adherencia al tratamiento: Se continua con los contenidos de la sesión anterior y se aclaran las dudas y dificultades encontradas. Se realizan ensayos conductuales de lo aprendido, mediante roleplaying en los que intervienen pacientes y familiares.

22. Regreso y adaptación a la comunidad:

- Componente de intervención cognitivo-conductual: Se ofrecen claves para la adaptación del paciente en la comunidad, si finalmente sufre una recaída. Se trabaja el manejo del estrés en la comunidad, elaborando planes y actividades diarias profesionales y recreativas. Se repasa la información del taller de empleo de la Sesión 16. Se debate sobre el consumo de sustancias y su relación con las recaídas, recomendando la adopción de conductas incompatibles con el consumo de sustancias e identificando hábitos de vida y actividades placenteras saludables.

- Se pide a pacientes y familiares que durante la semana anoten sus dudas e inquietudes para debatirlas en la próxima sesión.

23. Resolución de problemas:

- Resolución de las inquietudes de pacientes y familiares.

- Se consulta con los familiares los cambios percibidos en los pacientes y en sí mismos respecto al modo de afrontar la enfermedad.

- Se trabajan las habilidades sociales aprendidas mediante role-playing en los que participan pacientes y familiares.

- Se repasan técnicas como el reforzamiento positivo, modelado y moldeamiento, para que los familiares las lleven a cabo cuando sea necesario para ayudar al paciente respecto al cambio de conducta.

- Se administra el DAI, verificando si ha mejorado o no la adherencia a la medicación.

24. Satisfacción con el programa y cierre:

- Se comenta la evolución de los pacientes y los aspectos más relevantes de los autorregistros diarios y del DAl. Se analizan los resultados descriptivamente, incidiendo sobre los aspectos en los que el cambio sea menor. 
- Se enfatizan las percepciones de autoeficacia sobre los recursos adquiridos, las competencias desarrolladas y el manejo de la enfermedad, así como el funcionamiento en la vida diaria, de una forma holística.

- Se entrega a pacientes y familiares un cuestionario de evaluación final del programa, para que respondan si se han cumplido los objetivos propuestos, si la metodología era adecuada, si el tiempo empleado era suficiente y cómo podría mejorarse la intervención. Recomendamos realizar regularmente evaluaciones similares a lo largo de la intervención, o evaluaciones del proceso, con el fin de conocer la evolución de los participantes.

- Se entrega una escala de interés y satisfacción subjetiva, para que indiquen si el programa les ha gustado, si los contenidos les han resultado útiles y si ha mejorado su calidad de vida, así como su satisfacción general con la intervención.

- Se indica qué hacer en caso de experimentar una recaída y cómo buscar ayuda profesional. Se indica también cómo informar al equipo de la intervención actual de dicho evento.

- Se producen las despedidas y el cierre del programa.

\section{Discusión y conclusiones}

Una vez aplicado este programa, cabe esperar que se reduzcan las tasas de recaída (Eisner, Barrowclough, Lobban y Drake, 2014; Klingberg et al., 2010; Montaño, Nieto y Mayorga, 2013; Prasko et al., 2011) y la duración de la rehospitalización en caso de que se produzca (McKenna y Mortimer, 2014; Shean, 2007). Esto se debe a la mejora de la adherencia a la medicación (Montaño et al., 2013; Prasko et al., 2011; Shean, 2007) y al reconocimiento de los marcadores de recaída, permitiendo reaccionar ante ellos con la mayor prontitud y eficacia posibles (Eisner et al., 2014), evitando la exacerbación de los síntomas y estados clínicos más graves.

Asimismo, se espera que los pacientes incrementen su autoeficacia, gracias al fomento de recursos de autogestión de la enfermedad y de afrontamiento, así como al fortalecimiento de las creencias de los pacientes sobre su capacidad y eficacia para afrontar el estrés y los síntomas de la enfermedad (Eisner et al., 2014; Godoy et al., 2012).

Los beneficios no sólo repercuten en los pacientes, sino que también pretenden aliviar la carga de sus familiares, mejorando su calidad de vida y las relaciones familiares (Montaño et al., 2013; Shean, 2007).

Finalmente, al contrario de lo que ocurre con el tratamiento farmacológico, los beneficios del presente programa serían de larga duración y no presentarían efectos secundarios indeseables (Prasko et al., 2011), más bien al contrario: los recursos y competencias adquiridos por los participantes no sólo les ayudarán a manejar más adecuadamente la enfermedad sino a tener un mejor funcionamiento psicosocial en la vida diaria y, en general, un mayor bienestar y calidad de vida.

En cuanto a las limitaciones de la intervención, por su duración y extensión, los participantes deben mantener un alto grado de interés y motivación para adherirse adecuadamente a la misma. Consecuentemente, es necesario que cuenten con apoyo familiar y que su estado clínico sea estable. Además, sería recomendable que los grupos de trabajo fueran reducidos y se complementaran con sesiones individuales.

Ésta es una propuesta de intervención basada en la literatura y en la experiencia profesional de los autores, por lo que recomendamos la validación empírica de su 
utilidad y eficacia en términos tanto clínicos como personales en la futura investigación al respecto.

En definitiva, el programa favorecería el crecimiento de los recursos personales para la vida cotidiana y la autogestión de la enfermedad, ayudando a pacientes y familiares tanto en el manejo de la enfermedad como, en general, en la promoción de su salud, bienestar y calidad de vida.

\section{Referencias}

Álvarez-Jiménez, A., Priede, A., Hetrick, S. E., Bendall, S., Killackey, E., Parker, A. G., McGorry, P. D. y Gleeson, J. F. (2012). Risk factors for relapse following treatment for first episode psychosis: A systematic review and metaanalysis of longitudinal studies. Schizophrenia Research, 139, 116-128.

American Psychological Association, Society of Clinical Psychology (2013). Psychological treatments. Recuperado el 14 de Abril de 2015, de http://www.div12.org/psychological-treatments/treatments/.

American Psychiatric Association (2014). Guía de consulta de los criterios diagnósticos del DSM-5®: Spanish Edition of the Desk Reference to the Diagnostic Criteria From DSM-5®. Washington, DC: American Psychiatric Association.

Ascher-Svanum, H., Zhu, B., Faries, D. E., Salkever, D., Slade, E. P., Peng, X. y Conley, R. R. (2010). The cost of relapse and the predictors of relapse in the treatment of schizophrenia. BMC Psychiatry, 10, 1-7.

Britto, J. G. (2014). Relapse prevention among caregivers of patients with schizophrenia. Asian Journal of Nursing Education and Research, 4, 140-144.

Eisner, E., Barrowclough, C., Lobban, F. y Drake, R. (2014). Qualitative investigation of targets for and barriers to interventions to prevent psychosis relapse. BMC Psychiatry, 14, 1-14.

Godoy, J. F., Godoy-Izquierdo, D. y Vázquez, M. L. (2012). Programa de entrenamiento en autoeficacia para el afrontamiento del estrés en pacientes con esquizofrenia. En Valencia, M. (Ed.), Alternativas terapéuticas para la esquizofrenia (pp. 425-490). México: Herder.

Godoy, J. F., Muela, J. A. y Godoy-Izquierdo, D. (2012). Prevención de recaídas en la esquizofrenia. En Valencia, M. (Ed.), Alternativas terapéuticas para la esquizofrenia (pp. 73-137). México: Herder.

Godoy, J. F., Muela, J. A., Sánchez-Barrera, M. B., Sánchez-Huete, J. R. L., Pérez, M. y Lorite, A. (1995). Síntomas prodrómicos preepisodio/marcadores de vulnerabilidad en la predicción y prevención de recaídas en la esquizofrenia. Psicología Conductual, 3, 159-172.

Klingberg, S., Wittorf, A., Fischer, A., Jakob-Deters, K., Buchkremer, G. y Wiedemann, G. (2010). Evaluation of a cognitive behaviourally oriented service for relapse prevention in schizophrenia. Acta Psychiatrica Scandinavica, 121, 340-350.

Larson, M. K., Walker, E. F. y Compton, M. T. (2010). Early signs, diagnosis and therapeutics of the prodromal phase of schizophrenia and related psychotic disorders. Expert Review of Neurotherapeutics, 10, 1347-1359.

McKenna, P. J. y Mortimer, A. M. (2014). Current and future treatment modalities in schizophrenia: Novel antipsychotic drugs and cognitive therapy. Expert Review of Neurotherapeutics, 14, 67-73.

Montaño, L., Nieto, T. y Mayorga, N. (2013). Esquizofrenia y tratamientos psicológicos: Una revisión teórica. Revista Vanguardia Psicológica Clínica Teórica y Práctica, 4, 86-107.

Mueser, K. T. y Cook, J. A. (2014). Rising to the challenge of first episode psychosis: The NIMH Recovery After Initial Schizophrenia Episode (RAISE) Initiative. Psychiatric Rehabilitation Journal, 37, 267-269.

Mueser, K. T., Deavers, F., Penn, D. L. y Cassisi, J. E. (2013). Psychosocial treatments for schizophrenia. Annual Review of Clinical Psychology, 9, 465-497.

Olivares, J. M., Sermon, J., Hemels, M. y Schreiner, A. (2013). Definitions and drivers of relapse in patients with schizophrenia: A systematic literature review. Annals of General Psychiatry, 12, 1-11. 
Organización Mundial de la Salud. (2014). Esquizofrenia. Recuperado el 7 de Marzo de 2015, de http://www.who.int/mediacentre/factsheets/fs397/es/

Prasko, J., Vrbova, K., Latalova, K. y Mainerova, B. (2011). Psychoeducation for psychotic patients. Biomedical Papers of the Medical Faculty of Palacký University, Olomouc, Czech Republic, 155, 385-396.

Robles, R., Páez, F. y González, J. (2012). Terapia cognitivo-conductual para los trastornos psicóticos: Fundamentos, evaluación y aplicación a propósito de un caso. En Valencia, M. (Ed.), Alternativas terapéuticas para la esquizofrenia (pp. 331-361). México: Herder.

Samalin, L., Blanc, O. y Llorca, P. M. (2010). Optimizing treatment of schizophrenia to minimize relapse. Expert Review of Neurotherapeutics, 10, 147-150.

Shean, G. D. (2007). Recent developments in psychosocial treatments for schizophrenic patients. Expert Review of Neurotherapeutics, 7, 817-827.

Valencia, M., Liberman, R. P., Rascón, M. L. y Juárez, F. (2012). Habilidades psicosociales para la esquizofrenia. En Valencia, M. (Ed.), Alternativas terapéuticas para la esquizofrenia (pp. 23-71). México: Herder.

Velthorst, E., Koeter, M., van der Gaag, M., Nieman, D. H., Fett, A. K., Smit, F., Staring, A. B. P., Meijer, C. y de Haan, L. (2015). Adapted cognitive-behavioural therapy required for targeting negative symptoms in schizophrenia: Meta-analysis and meta-regression. Psychological Medicine, 45, 453-465. 